\title{
VIOLENCIA Y VIOLENCIA SIMBÓLICA EN LA MAQUILA EN CIUDAD DEL ESTE
}

Victor Hugo Jara Cardozol

\begin{abstract}
RESUMEN: El objetivo principal del presente artículo es describir las relaciones simbólicas que intermedian el contexto laboral maquilador y los/las trabajadores/as de maquilas en Ciudad del Este (Paraguay). Partiendo del concepto de violencia simbólica de Bourdieu, comprendemos que los actores sociales se encuentran inmersos en campos llenos de significados que permiten/determinan las relaciones de poder, por ello, se hará una descripción detallada tanto del contexto laboral maquilador como de los/las trabajadores/as para comprender sus determinantes estructurales y simbólicas. Realizamos el trabajo principalmente a partir de entrevistas semiestructuradas a trabajadores de maquiladoras textiles. La técnica de muestro utilizada fue bola de nieve. Concluimos que el contexto laboral de las maquilas es un espacio hostil con precarias condiciones de trabajo afectando negativamente a la clase trabajadora.
\end{abstract}

Palabras claves: Paraguay; Maquila; Violencia; Bourdieu.

\section{VIOLÊNCIA E VIOLÊNCIA SIMBÓLICA NA MAQUILA DA CIUDAD DEL}

\section{ESTE}

RESUMO: O objetivo principal deste artigo é descrever as relações simbólicas que medeiam o contexto de trabalho maquiladora e as trabalhadoras maquiladoras de Ciudad del Este (Paraguai). Partindo da concepção de violência simbólica de Bourdieu, entendemos que os atores sociais estão imersos em campos carregados de significados que permitem / determinam as relações de poder, portanto, será feita uma descrição detalhada tanto do contexto de trabalho maquiladora quanto dos trabalhadores. compreender seus determinantes estruturais e simbólicos. Realizamos o trabalho principalmente a partir de entrevistas semiestruturadas com trabalhadores de maquiladoras têxteis. A técnica de amostragem utilizada foi bola de neve. Concluímos que o contexto de trabalho das maquiladoras é um espaço hostil com condições precárias de trabalho afetando negativamente a classe trabalhadora.

Palabras claves: Paraguay; Maquila; Violencia; Bourdieu.

\section{INTRODUCCIÓN}

\footnotetext{
${ }^{1}$ Formado em Ciencia Politica e Sociologia na Universidade Federal da Integracao Latinoamericana, atualmente mestrando no PPG em Sociedade Cultura e Fronteiras. Tenho experiencia na area de Sociologia do Trabalho. E-mail: junior_jara18@hotmail.com 
La industria maquiladora de exportación nació a mediados de la década del 1960 en la frontera entre Estados Unidos y México en el marco de un gran proceso de reestructuración de la economía mundial. Según Cuero (2018, p.144), la maquila forma parte de un "esquema de subcontratación internacional" que "se dio en medio de una crisis del patrón de acumulación fordista y de la instauración de una nueva fase de la división internacional del trabajo.”

Básicamente, consiste en el desplazamiento de la parte de ensamblaje de la cadena de producción de una mercancía. Esta compartimentación de la línea de montaje es la versión internacional de la tercerización, donde capitales buscan principalmente a países con fuerza de trabajo barata y una legislación laboral flexible para, o bien, construir una filial de su empresa, o bien, contratar los servicios de una planta maquiladora (ANTUNES, 1999, p. 56). Esto ocurre en el contexto de la crisis desencadenada en la década de 1970 (CASTILLO \& SOTELO, 2013), que llevó al modo de producción capitalista a una crisis estructural, siendo la "caída tendencial de la tasa de ganancia" la expresión más contundente de este proceso (AUNTUNES, 2017, p. 33); (CARCANHOLO, 2008); (ALVES, 2018, p. 75).

El germen de la maquila se encuentra en el Programa de Industrialización Fronteriza (PIF), una propuesta realizada para hacer frente a las altas tasas de desempleo generadas por la finalización del programa braceros, que a su vez, fue un programa de contratos temporales para migrantes que tuvo lugar entre 1942 y 1964 (DURAND, 2007) que consistía en la contratación de trabajadores mexicanos en los campos del sur de Estados Unidos que, por causa de la primera guerra mundial, carecía de "brazos" para trabajar los campos.

Luego de más 50 años la industria maquiladora en México ha mostrado cierto nivel de desarrollo tecnológico (Contreras y Munguía, 2007), sin embargo, aún continúa siendo mayoritariamente una industria que utiliza fuerza de trabajo poco especializada envuelta en actividades que requieren alto componente físico (CUERO, 2018, p. 145).

Varios estudios en México y en países de Centroamérica, han denunciado y analizado las precarias condiciones de trabajo que ofrecen las maquiladoras (Berrueta, 2010); (CECEÑA, 1992); (OXFAM, 2015); (FIDH, 2005); (CDM y CHAAC, 2009).

Baumgratz y Cardin (2019, p. 82), señalan que: 


\begin{abstract}
Os empregadores buscam um perfil de funcionário de pessoas submissas, dispostas a acatar qualquer ordem, sem horários definidos, com salários baixos e sem plano de saúde. Destarte, existe a conhecida e temida "lista negra", onde os nomes dos funcionários "revoltosos" -aqueles que exigem algum direitosão compartilhados entre as empresas, para impedir a contratação de funcionários "causadores de problemas.".
\end{abstract}

En Paraguay, se han abierto las puertas a las maquiladoras en el año 2000, a partir de la reglamentación de la ley $\mathrm{N}^{\circ} 1.064 / 97$. Y si bien, ya han pasado más de 20 años desde su presencia en el país, ésta recién ha comenzado a ser significativa a partir del $2013^{2}$.

Amén de la relativa corta vida de la maquila en Paraguay, no existe mucha bibliografía sobre el tema, y de entre la que existe, ninguna se ha debruzado a analizar detenidamente la situación de la clase trabajadora.

Precisamente, son los medios de comunicación los que nos han informado de denuncias de trabajadores de maquilas en Ciudad del Este sobre precarias condiciones de trabajo y alevosos incumplimientos de la legislación laboral vigente (La clave, 2019), (Vanguardia, 2019). A pesar de esta clase trabajadora representar más de 6.000 puestos de trabajo (CNIME, 2019), no se han visto organizaciones gremiales de ningún tipo que pudieran dar un ropaje organizado a sus denuncias.

Sin embargo, esta no es una situación propia de Paraguay, también es característica de algunos puntos importantes del territorio maquilador en México, como lo menciona Berruta (2010) en el caso de Ciudad Juarez, México. El autor sostiene que la Maquila, en la medida del crecimiento de su dimensión espacial, ha redefinido el territorio fronterizo "llegando a determinar prácticamente en su totalidad el funcionamiento social y cultural de todo el territorio" (BERRUTA, 2010 p. 31), lo que ha generado una sociedad desestructurada como consecuencia de la violencia simbólica y estructural que supone el proyecto económico de la maquila (BERRUTA, 2010, p. 36).

El presente trabajo reflexiona sobre la violencia simbólica en el territorio maquilador textil y cómo estas afectan a la fuerza de trabajo. Para ello, utilizaremos el concepto de "violencia simbólica" de Pierre Bourdieu para comprender las dinamias sociales que se tejen entre el espacio laboral maquilador y los/las trabajadores/as.

\footnotetext{
${ }^{2}$ Desde el 2001 hasta el 2012 se habían aprobado 44 proyectos de maquiladoras, y entre 2013 y 2018, se aprobaron un total de 136 proyectos (CNIME, 2019) 
Así, en total, se entrevistaron a 22 personas, de las cuales, 7 fueron grabadas y transcritas: 3 trabajadores/extrabajadores de la planta maquiladora textil seleccionada, una trabajadora de una planta maquiladora de auto partes, un abogado laboralista, 14 vendedores del centro comercial de Ciudad del Este, 2 dirigentes de la Central Unitaria de Trabajadores Auténtica (CUT-A), y una dirigente del Sindicato de funcionarios/funcionarias y educadores/as populares de la Secretaria Nacional de la niñez y adolescencia (SIFEPOSNNA).

Es necesario mencionar que la presente propuesta no plantea una caracterización completa y acabada de la clase trabajadora de la maquila, sino más bien un acercamiento reflexivo que ayude a cimentar investigaciones sociológicas posteriores que consigan dar cuenta de una caracterización completa de la problemática, esto debido principalmente a que creemos que el tamaño de la muestra no es suficientemente representativo, lo que, sin embargo, no nos impide realizar estas aproximaciones reflexivas a la problemática planteada.

Las demás fuentes son los datos estadísticos del Ministerio de Industria y Comercio (MIC), el Consejo Nacional de la Industria Maquiladora de Exportación, artículos científicos y disertaciones de maestrías.

El objetivo general es describir las relaciones simbólicas que se tejen entre el espacio maquilador y el/la trabajador/a, para lograr ello con mayor claridad, se analizará en un primer momento a la industria maquiladora, su contexto y sus relaciones con el mercado nacional e internacional; luego, a partir de las entrevistas a trabajadores, se analizarán las condiciones de trabajo, así como el contexto más general de la reproducción de sus vidas; para así, finalmente, analizar la relación que se tejen entre ambos sectores.

\section{LA INDUSTRIA MAQUILADORA, UNA EXTENSIÓN DEL COMERCIO MUNDIAL}

Así como mencionado en la introducción, la maquila nace en el marco de la reestructuración del capitalismo internacional como consecuencia de una crisis estructural que desnuda un sistema económico desgastado que ya no consigue sostenerse en los moldes adquiridos posterior a la segunda guerra mundial. 


\begin{abstract}
El programa de maquila es el resultado de las intrincadas relaciones sociales, económicas y políticas entre México y Estados Unidos, en el contexto de una creciente competencia global y un rápido cambio tecnológico. (...) La maquila debe entenderse como un fenómeno perteneciente a la reorganización internacional de la producción en la segunda mitad del siglo XX, como bien se describe en la teoría del ciclo del producto. (BUITELAAR \& PADILLA, 2000 p. 1628$)^{3}$.
\end{abstract}

En el marco de esta reestructuración, el desarrollo del capitalismo japonés, marcado principalmente por un nuevo paradigma organizacional de la producción toyotismo-, deja en evidencia las falencias del fordismo obligando a la industria norteamericana y europea a adaptarse a la productividad que la competencia japonesa estaba exigiendo.

El toyotismo se había basado principalmente en un proceso productivo más "flexible", donde el trabajador deja de ser un simple apéndice de la máquina inmerso en un proceso productivo vertical a ser un trabajador multifuncional en una estructura pretendidamente más horizontalizada. En vez de la producción en masa de mercancías estandarizadas del fordismo, la producción buscó adaptarse más y mejor a las exigencias del mercado, aumentado la diversidad y adecuando la cantidad exactamente a la demanda. Parte de este proceso también fue la disminución de trabajadores dentro la fábrica principalmente a través de dos formas: primera, mediante el aumento de funciones realizadas por los trabajadores y, segundo, mediante la tercerización de la producción.

Este proceso de descentralización es, tal vez, la principal característica que luego tendrá enormes repercusiones en el escenario internacional. Según Antunes (2017, p. 226227 ) “Enquanto na fábrica fordista cerca de $75 \%$ era produzido no seu interior, na fábrica toyotista somente cera de $25 \%$ é produzido no seu interior. Ela horizontaliza o processo produtivo e transfere a "terceiros" grande parte de que anteriormente era produzido dentro dela".

En ese sentido, las grandes firmas ubicadas en los países centrales del capitalismo mundial, comienzan a descentralizar algunas partes del proceso productivo hacia países con oferta de fuerza de trabajo más barata para así dar respuesta a la competencia internacional.

\footnotetext{
${ }^{3}$ Cita original en inglés. 
En respuesta, las empresas manufactureras estadounidenses miraron a México y la Cuenca del Caribe como su reserva de mano de obra menos calificada para contrarrestar el desafío asiático y mover hacia el sur sus procesos de producción intensivos en mano de obra. (Frobel, Heinrichs \& Kreye, 1980; Grundwald \& Flamm, 1985) (BUITELAAR \& PADILLA, 2000, 1630) ${ }^{4}$.

La planta maquiladora, por regla general, tiene casi un nulo contacto con la economía del país donde se encuentra, ya que, además de las numerosas exenciones impositivas que recibe, nada de la maquinaria ni materia prima es proveída por el mercando nacional, sino que es importada de la matriz, y así mismo, una vez terminado el proceso de ensamble, toda la producción ${ }^{5}$ es reexportada.

El principal discurso de las autoridades de los países que albergan a las maquilas es que esta es una oportunidad para estimular el desarrollo industrial, arguyendo que este tipo de interacción con la cadena global de producción podría generar transferencia de tecnología y una fuerza de trabajo más capacitada.

Sin embargo, se ha constatado que ella no ha generado un importante impacto en la economía nacional de los países que la reciben más allá de los salarios que deja a los/as trabajadores/as. Es, en suma, una modalidad productiva más ligada a las vicisitudes del mercado internacional que a las del contexto doméstico nacional, determinando también, de esta forma, las condiciones de trabajo ofrecidas a la clase trabajadora.

De esta forma, la clase trabajadora por primera vez se encuentra en una situación de competencia internacional, compitiendo unas con otras estirando los salariaos para abajo, así como precarizando las condiciones de trabajo. En Honduras se ha llegado, incluso, a legislar un "salario mínimo diferenciado" para las maquilas, uno menor que el salario mínimo nacional, como una forma de "incentivo para generar empleo" en regiones categorizadas como "deprimidas económicamente" (HONDURAS, p.47)

\section{CONDICIONES DE TRABAJO Y CONTEXTO DE LA CLASE TRABAJADORA}

\footnotetext{
${ }^{4}$ Cita original en inglés.

${ }^{5}$ En el caso paraguayo, es posible comercializar un $10 \%$ de la produccion en el mercado nacioal previa aotoriazación del Consejo Nacional de la Industria Maquiladora de Exportación (CNIME). 
En el presente apartado, analizaremos a la clase trabajadora en dos espacios de producción: primero, en el contexto más general de la reproducción de su vida fuera del espacio laboral y, segundo, las condiciones de trabajo.

\section{La clase trabajadora esteña}

La reproducción de la vida del trabajador promedio de clase media baja en Ciudad del Este es precaria. Según el boletín trimestral de empleo de la DGEEC, del segundo trimestre del 2019, a nivel nacional, el porcentaje de asalariados (públicos y privados) que ganan menos que salario mínimo es de 34,1 \% (DGEEC, 2019, p. 17), así mismo, en el departamento de Alto Paraná, el desempleo o sub empelo, que, combinados -tasa de desempleo y sub empleo por insuficiencia de trabajo- alcanzan el $10 \%$ de la población del departamento del Alto Paraná, un total de 40.830 personas; y además, donde la pobreza total y extrema, combinadas, afectan al $24,5 \%$ de la población, un total de 197.528 personas (DGEEC, 2018).

La gran mayoría que se encuentra trabajando, no tiene condiciones de estabilidad plena debido a que el mercado laboral es bastante "flexible", expulsando y contratando constantemente, manteniendo a la clase trabajador en una constante "circulación".

\footnotetext{
Contudo, com decorrência desses processos, um bom índice a se observado é a diminuição do tempo médio de permanência no emprego em todos os países que produzem dados a respeito. Da mesma forma, também é significativo o declínio ou praticamente o fim do chamado "trabalho para toda a vida", mesmo nos países que, por exemplo, foram caracterizados por grupos de trabalhadores que por gerações ao fio apresentavam circulação praticamente nula da força de trabalho. (GIL, 2018, p. 128).
}

Esta pérdida de estabilidad y aumento de la circulación genera que el trabajador empleado, bajo la constante amenaza del desempleo, sea un desempleado potencial en todo momento. Esto genera que la diferencia entre empleo y desempleo tienda a confundirse generando una situación bastante inestable lo que además causa, evidentemente, mucha incertidumbre.

A esta situación, la precariedad de los servicios básicos públicos en general completa este cuadro de precarización estructural en la que el trabajador se encuentra. Las escuelas públicas sólo tienen funcionamiento medianamente aceptable en los centros 
urbanos. En la medida en que las escuelas se ubican a más distancia, se pueden ver escuelas sumidas en la desidia total, haciendo muchas veces que sea más seguro y salubre dar clases bajo los árboles que dentro de las salas de aula.

Así mismo, el sistema de salud y de transporte muestran un cuadro similar. Los hospitales muestran, desde una estética que evidencia la falta de mantenimiento y cuidados de salubridad, hasta el constante desabastecimiento de insumos básicos como jeringas o algodón. Los colectivos del transporte público en Paraguay, por sí solos, podrían permitir fecundos análisis sobre la realidad de la clase trabajadora. Vehículos con más de cuatro décadas de antigüedad, sin los más mínimos cuidados de seguridad interna ni externa, condiciones de mecánicas rozando lo criminal constituyen el pan de cada día de un usuario.

Como vemos, el contexto de la reproducción de la vida de la clase trabajadora reviste un cuadro bastante precario que, claramente, tiene efectos directos en la construcción de la conciencia colectiva e individual de los mismos. La precariedad de su entorno es naturalizada en su habitus, ella forma parte esencial del conjunto de estructuras simbólicas que le dan sentido y estructuran su comportamiento individual y su interacción con la sociedad.

\section{Las condiciones de trabajo en la planta maquiladora}

Uno de los principales aspectos referidos por nuestros entrevistados, tiene que ver con las elevadas exigencias de productividad, haciendo con que el trabajo sea realizado con niveles muy elevados de intensidad. Estos niveles de productividad son posibles gracias a dos mecanismos de incentivos: el económico y el amedrentamiento.

\section{Incentivos}

Ambas formas de incentivos, funcionan como mecanismos que estimulan, aumentan y mejoran la producción. Si bien, según los relatos, ambos mecanismos están siempre presentes, en algunos lugares uno es más importante que el otro, y viceversa.

La forma de los incentivos depende del contexto de la fábrica y de las posibilidades de aplicar una u otra forma. Por lo general, aquellas maquiladoras que 
utilizaban el incentivo económico eran aquellas que tenían vínculos más estrechos con las multinacionales, como el caso de la maquila de Adidas, y esto, a su vez, debido a que la multinacional exigía el cumplimiento estricto de la normativa laboral del país, enviando constantemente fiscalizadores que sancionaban si encontraban incumplimientos.

Estos incentivos (los económicos), conseguían que los trabajadores llegasen a trabajar incluso 12 horas en un día sin que los mismos reclamasen, todo lo contrario, como las horas extras eran pagas, los trabajadores veían con buenos ojos esa extensión de la jornada de trabajo.

Por otro lado, el amedrentamiento, la violencia -y el miedo como principal consecuencia- es un tipo de incentivo propio de modos de organización de trabajos más precarios, materializándose en tratos hostiles desde la patronal: gritos, amenaza constante de despido, descuentos compulsivos.

En varios talleres, si la meta no es alcanzada, los trabajadores no pueden salir de la fábrica. La jornada de trabajo, en promedio, es de 9 horas y media por día, de lunes a viernes, de 6:00 a 16:30. Si, llegado el horario, estos aún no hubieren llegado a la meta, y aún así se retiran, se les castiga con un día de suspensión y con el descuento de la jornada correspondiente. Una forma de violencia simbólica es realizada a través de reuniones entre todos los trabajadores de la fábrica con el gerente.

En las reuniones, el gerente expone sus críticas a los trabajadores frente a toda la fábrica. Ser objeto de crítica en ese espacio genera el miedo suficiente como para hacer todo lo posible para evitarlo. Refiriéndose a su experiencia en una planta maquiladora, un entrevistado manifestó que “(...) ahí manda el miedo, hasta ahora sigue mandando el miedo".

Además del hecho de que las horas extras en este tipo de talleres no son pagadas, había casos de trabajadores de confección de sábanas se quedaban trabajando desde las 06:00 hasta las 23 hs de la noche, constituyendo así una jornada de trabajo de 17 horas.

Los principales aspectos de la territorialidad en los talleres maquiladores buscan estimular la competencia entre trabajadores, minando los sentimientos de solidaridad y fraternidad. En suma, son políticas de asilamiento, lo que suele generar, en ocasiones, ambientes laborales marcados por una profunda hostilidad entre compañeros y compañeras de trabajo. 


\section{Inestabilidad}

Otra de las características que atraviesa a las maquilas es el de la inestabilidad. Estas plantas pueden llegar a migrar con una enorme facilidad, dejando a miles de trabajadores en la calle de la noche para la mañana. Por otro lado, si bien, como hemos apuntado, la industria maquiladora ha comenzado recién a tener importancia hace aproximadamente 10 años, este ha sido un tiempo suficiente para constatar la gran inestabilidad de los puestos de trabajo en las maquilas.

Uno de nuestros entrevistados trabajó en una de los primeros talleres textiles instalados en la región, en el 2008 -incluso antes de que el sector comenzara a tener importancia-. En 10 años de trabajo, hasta el 2018, pasó por 7 plantas maquiladoras, en 4 de ellas superó a penas el mes de trabajo.

\section{Circulación de un trabajador}

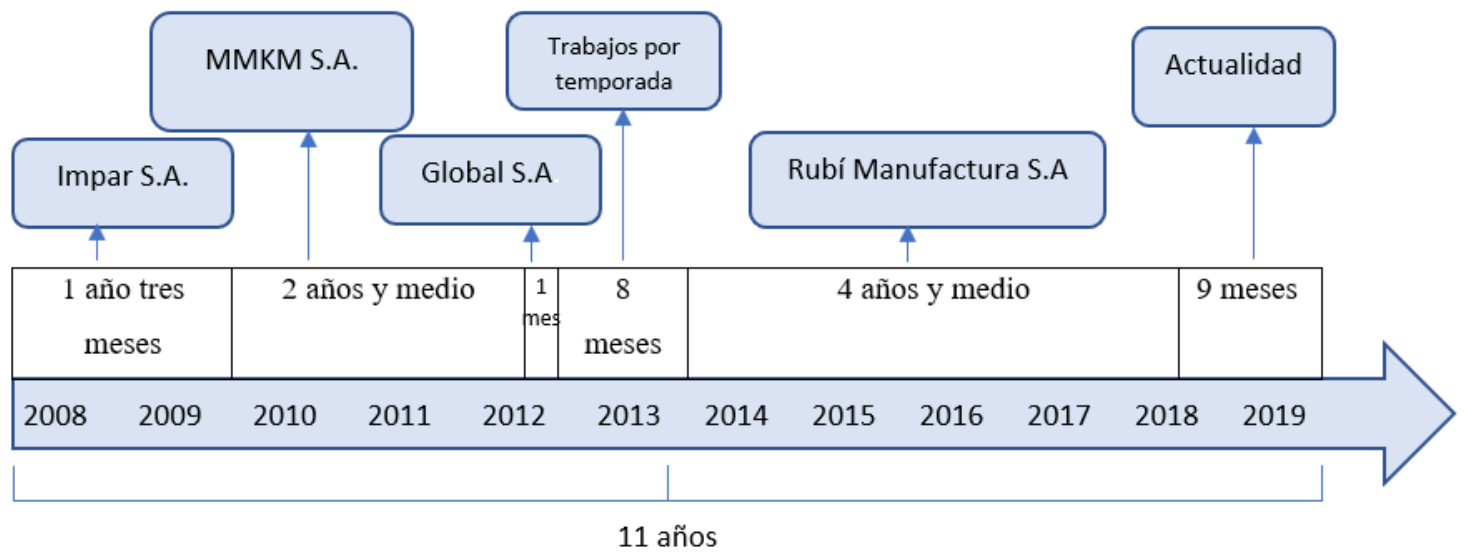

Figura 3

Fuente: elaboración propia a partir de una entrevista con un trabajador

Hemos elaborado este pequeño diagrama para mostrar con mayor claridad lo recién mencionado. En el año de 2013, estuvo 8 meses realizando trabajos por temporada en tres talleres distintos. Como la vinculación de la demanda es netamente con la matriz, las plantas maquiladoras trabajan bajo pedidos que suelen tener importantes variaciones, llegando a contratar y despedir hasta a 100 trabajadores en un lapso de 2 a 3 meses.

\section{DISCUSIÓN}


Para Bourdieu, tanto la violencia como la dominación simbólica, están determinadas por un conjunto de estructuras simbólicas propias del individuo o grupo que se encuentra ejerciendo la violencia y/o dominación y del/los que la padece/n, así como la del espacio donde se reproduce el sometimiento simbólico. Esencialmente, su concepto excluye la violencia física y verbal explícita, remitiéndose a interacciones "más finas", difíciles de percibir y sentir, en la medida en que se encuentran ya naturalizadas por los habitus de los/as que interactúan, requiriendo ciertas "complicidades", incluso, de la persona sometida y/o violentada.

\begin{abstract}
No es ya tan falso decir que la causa de la timidez reside en la relación entre la situación o la persona intimidante (que puede negar la conminación que dirige) y la persona intimidada; más exactamente, entre las condiciones sociales de producción de ambas. Lo que acaba remitiendo a toda la estructura social. (Bourdieu, p, 25).
\end{abstract}

A esto, agrega que

Todo hace suponer que las instrucción más determinantes para la construcción del hábitus se transmiten sin pasar por el lenguaje y la conciencia, a través de sugestiones inscritas en los aspectos aparentemente más insignificantes de las cosas, de las situaciones o de las prácticas de la existencia común: así, la modalidad de las prácticas, las maneras de mirar, de comportarase, de guardar silencio e incluso de hablar (miradas desaprobadoras, tonos o aires de reproche, etc.) están cargados de conminaciones. (Bourdieu, p, 25).

Los campos, para Bourdieu, son espacios de socialización que rivalizan con otros campos en relaciones de poder, dominación y dependencia, marcados, principalmente, por capitales, a saber: el económico, el social y cultural. La pertenencia a un campo con cierto capital, se evidencia de diversas formas: la ropa, el acento, el tono de voz, el acento al hablar, la forma de caminar. Todos estos elementos delatan la procedencia del grupo o individuo. Tal es así que, "La relación entre dos personas puede ser tal que basta con que aparezca una para que inmediatamente imponga a la otra sin que ni siquiera sea necesario que la primera lo desee, menos aún que lo ordene (...)” (Bourdieu, p. 28). En el momento del encuentro, se activan las estructuras simbólicas de los habitus en interacción posicionándose cada uno de acuerdo a su ubicación jerárquica social. 
De esta forma, para comprender mejor las interacciones entre seres sociales es necesario conocer el espacio de reproducción de cada uno. Solo conociendo las variables materiales y objetivas, así como las simbólicas, que forman parte del espacio de producción, utilización y reproducción de los respectivos campos, se podrá comprender mejor cómo interactúan.

Como hemos visto, la reproducción del trabajador se encuentra plagado de situaciones de violencia tanto manifiesta como simbólica. Al convertirse la incertidumbre en un aspecto "natural" de su vida cotidiana, busca extender lo máximo posible los momentos de estabilidad, aunque ello sea a costa de precarias condiciones laborales.

A pesar de la industria maquiladora ser caracterizada por nuestros entrevistados por condiciones bastante precarias, donde a la intensidad de la labor, se le suma la precariedad de las instalaciones -llegando a representar riesgos para la salud-, horas extras no pagadas, situaciones de hostilidad emocional y psicológica, para muchos, la Maquila constituye la oportunidad de ganar un salario mínimo por primera vez.

Esta surte de resignación con la precariedad, es condición fundamental de la instalación de las plantas maquiladoras, ya que, debido a la intensa competencia internacional y a la necesidad de reducir costos a niveles competitivos, estas se abalanzan en busca de fuerza de trabajo barata "explotable".

El sometimiento del trabajo a las necesidades de reproducción del capitalismo mundial necesariamente desvirtúa su esencia convirtiéndola en herramienta de sometimiento del trabajador, "Isso porque sob o sistema de metabolismo social do capital, o trabalho que estrutura o capital desestrutura o ser social." (ANTUNES, 2017, p. 261).

\section{COMENTARIOS FINALES}

La maquila forma parte de una de las estrategias de adaptación del capitalismo a las recurrentes crisis y a las exigencias de la competencia internacional. Hemos visto que en su esencia misma se encuentra la necesidad de reducir costos, lo que ha repercutido en las muy precarias condiciones de trabajo que ofrece.

El territorio maquilador, al encontrarse atravesado por estas determinaciones estructurales que lo definen, se erige como un espacio hostil, violento y desarticulador de los/as obreros/as que en ella trabajan. La interacción constante con la precariedad de la 
reproducción de la vida privada con la violencia manifiesta y simbólica imposibilita la crecimiento de sentimientos colectivos capaces de germinar en una "conciencia de clase" y así exigir mejores condiciones laborales de forma organizada.

Esa situación de aislamiento colectivo en ocasiones genera crudas competencias entre los/as trabajadores/as para mantener sus espacios transitorios de estabilidad.

Partiendo del presupuesto de que es necesario comprender el espacio de producción, reproducción y utilización, en una palabra, del contexto más amplio de los actores sociales para luego, primero, comprender mejor las determinaciones estructurales que la atraviesan y así, el espacio simbólico que lo recubre de significado, para después, segundo, comprender mejor la interacción entre ambos polos del análisis.

\section{REFERENCIAS BIBLIOGRÁFICAS}

ALVES, Gioavanni. A superexploração do trabalho e o colapso/expansão da formavalor no capitalismo global: notas teóricas. In: GIL, Felix \& GUANAIS, Juliana (org.). Superexploração do trabalho no século XXI. 1.ed. São Paulo: Projeto Editorial PRAXIS, 2018. pp. 69-94.

ANTUNES, Ricardo. Os sentidos do trabalho: Ensaio sobre a afirmação e a negação do trabalho. 2.ed. São Paulo: BOITEMPO, junho 2017.

BAUMGRATZ, D.; CARDIN, E. G. O regime de maquila e suas implicações no México: perspectivas para o modelo adotado no Paraguai. Estud. int. (Santiago, en línea) vol.51 no.192 Santiago abr. 2019. versión On-line ISSN 0719-3769. Disponible en: $<$ https://scielo.conicyt.cl/scielo.php?script=sci_arttext\&pid=S0719-

37692019000100071\&lang=es\#aff1 > . Acceso en: 08 de octubre 2020.

BERRUTA, Luis Humberto Méndez. Territorio maquilador y violencia. El caso de Ciudad Juárez. El Cotidiano, núm. 164, noviembre-diciembre, 2010, pp. 27-40. Universidad Autónoma Metropolitana Unidad Azcapotzalco. Distrito Federal, México. ISSN: 0186-1840. Disponible en: <http://www.redalyc.org/articulo.oa?id=32515894005 $>$. Acceso en :10 de octubre 2020.

BUITELAAR, Rudfolf \& PADILLA Pérez, Ramón. Maquila, Economic Reform and Corporate Strategies. Elsevier Science World Development. Gran Bretaña. Vol. 28, No. 9, pp. 1627-1642, 2000. Disponible en: http://directory.umm.ac.id/Data\%20Elmu/jurnal/UVW/World\%20Development/Vol28.I ssue9.Sep2000/1047.pdf. Acceso en 14 de abril 2021.

CARCANHOLO, Reinaldo. Aspectos teóricos de la crisis capitalista. Rebelión. 2008. Disponible en: https://rebelion.org/aspectos-teoricos-de-la-crisis-capitalista/. Acceso en: 14 abril 2021. 
CASTILLO Fernandez, Dídimo; SOTELO Valencia, Adrián. Outsourcing and the New Labor Precariousness in Latin America. Latin American Perspectives Issue 192, Vol. 40 No. 5, September 2013 14-26. DOI: 10.1177/0094582X13492124. Disponible en: https://journals.sagepub.com/doi/abs/10.1177/0094582x13492124. Acceso en 10 de Jun. 2021.

CECEÑA, Ana Esther. MAQUILADORAS Y TLC: dos expresiones de una problemática. Ciudad de México: Revistas Unam, v. 28, n. 88, 1992. Disponible en <http://www.revistas.unam.mx/index.php/pde/article/view/34490>. Acceso: 01 mayo 2018. Acceso en :06 de octubre 2020.

CEMAP. CAMARA DE EMPRESAS MAQUILADORAS DEL PARAGUAY. Asunción: Cemap, 2017.Disponible en: < http://www.maquila.org.py/?p=422>. Acceso: 01mayo. 2018.

CUERO, Julieta Martínez. La subcontratación como estrategia de rentabilidad para el capital transnacional: la industria maquiladora en Tijuana, 1990-2017. Anál. econ. vol.33 no.84 Ciudad de México sep./dic. 2018. versión On-line ISSN 2448-6655versión impresa ISSN 0185-3937. Disponible <http://www.scielo.org.mx/scielo.php?script=sci_arttext\&pid=S2448-

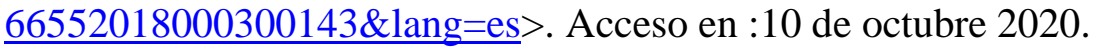

CONTRERAS Ó. F.; MUNGUÍA L. F.. Evolución de las maquiladoras en México. Política industrial y aprendizaje tecnológico. Región y sociedad vol.19 spe Hermosillo ene. 2007. versión impresa ISSN 1870-3925. Disponible en: http://www.scielo.org.mx/scielo.php?script=sci_arttext\&pid=S1870$\underline{39252007000400005}$ >. Acceso en :06 de octubre 2020.

CENTRO DE DERECHOS DE MUJERS (CDM); COALICIÓN HONDUREÑA DE ACCIÓN CIUDADANA (CHAAC). Impacto del libre comercio en los derechos laborales de las obreras de la maquila textil en honduras. Dirección de estudio y elaboración de infrome: Yadira Minero Rodas. Disponible en <http://www.corteidh.or.cr/tablas/29031.pdf> Acceso en: 12 Jun. 2019.

DURAND, Jorge. EL PROGRAMA BRACERO (1942-1964): UN BALANCE CRÍTICO. Migración y desarrollo, México, n. 9, p. 27-43, segundo semestre 2007. Disponível em <https://www.redalyc.org/pdf/660/66000902.pdf> accedido em 27 jul. 2021. ISSN: 1870-7599.

DIRECCIÓN GENERAL DE ESTADÍSITICAS, ENCUESTAS Y CENSOS (DGEEC). Principales resultados de Pobreza Monetaria y distribución de ingresos 2018. Asunción, 2018. Disponible en:

<https://www.dgeec.gov.py/Publicaciones/Biblioteca/POBREZA-MONETARIA\%202018/Pobreza\%20Monetaria_Boletin.pdf>Acceso en: 24 Jun. 2019>. 
DIRECCIÓN GENERAL DE ESTADÍSITICAS, ENCUESTAS Y CENSOS (DGEEC). BOLETÍN TRIMESTRAL DE EMPLEO, 1er trimestre 2019. Asunción. Mayo, 2019. Disponible en:

<https://www.dgeec.gov.py/Publicaciones/Biblioteca/EPHC2019/Primer\%20Trimestre/ Boletin\%20trimestral\%20de\%20empleo\%20EPHC_T1_2019.pdf > Acceso en: 24 Jun. 2019.

FEDERACIÓN INTERNACIONAL DE LOS DERECHOS HUMANOS fidh. Misión internacional de investigación. Situación de los derechos humanos en la maquilas de america central. $\mathrm{N}^{\circ} \quad 428 / 3$ Octubre 2005. Disponible en <https://www.fidh.org/IMG/pdf/maq427.pdf> Acceso en: 12 Jun. 2019, 02:11

GIL, Felix. Circulação e superexploração do trabalho: agenda de estudos da condição proletária contemporânea. In: GUANAIS, J.; FELIX, G. (Org) Superexploração do trabalho no século XXI: debates contemporâneos. São Paulo: Projeto editorial PRAXIS, 2018. Páginas (127-59).

OXFAM INTERMON. DERECHOS QUE PENDEN DE UN HILO. Zonas francas textiles frente a cooperativas de comercio justo. ABRIL 2015. Disponible en: $<$ https://oxfamintermon.s3.amazonaws.com/sites/default/files/documentos/files/Derecho QuePendenDeUnHilo.pdf> Acceso en: 12 Jun. 2019. 